OPEN ACCESS

Edited by:

Ming $L i$,

Kunming Institute of Zoology, China

Reviewed by:

Xingguang Luo,

Yale University, United States

XiongJian Luo,

Kunming Institute of Zoology, China

Li Hui,

Suzhou Guangji Hospital, China

Chen Zhang,

Shanghai Jiao Tong University, China

*Correspondence:

Xiaohong Gong

gongxh@fudan.edu.cn

Fei Wang

fei.wang@yale.edu

Yanqing Tang

tangyanqing@cmu.edu.cn

Specialty section:

This article was submitted to

Neurogenomics,

a section of the journal

Frontiers in Neuroscience

Received: 19 March 2021

Accepted: 31 May 2021

Published: 04 August 2021

Citation:

Tang L, Liu J, Zhu Y, Duan J, Chen Y, Wei $Y$, Gong $X$, Wang $F$ and Tang $Y$ (2021) ANK3 Gene Polymorphism Rs 10994336 Influences Executive Functions by Modulating Methylation in Patients With Bipolar Disorder. Front. Neurosci. 15:682873. doi: 10.3389/fnins.2021.682873

\section{ANK3 Gene Polymorphism Rs10994336 Influences Executive Functions by Modulating Methylation in Patients With Bipolar Disorder}

\author{
Lili Tang ${ }^{1}$, Juan Liu ${ }^{1}$, Yue Zhu ${ }^{1}$, Jia Duan ${ }^{1}$, Yifan Chen ${ }^{1}$, Yange Wei ${ }^{2}$, Xiaohong Gong ${ }^{3 *}$, \\ Fei Wang ${ }^{1,2 *}$ and Yanqing Tang ${ }^{1 *}$ \\ ${ }^{1}$ Department of Psychiatry, The First Affiliated Hospital of China Medical University, Shenyang, China, ${ }^{2}$ Early Intervention Unit, \\ Department of Psychiatry, Affiliated Nanjing Brain Hospital, Nanjing Medical University, Nanjing, China, ${ }^{3}$ State Key Laboratory \\ of Genetic Engineering and Human Phenome Institute, School of Life Sciences, Fudan University, Shanghai, China
}

Background: A large body of evidence suggests that epigenetic modification including DNA methylation plays a critical role in BD's pathogenesis while the identification of methylation quantitative trait loci (meQTLs) shed light on the interpretation of the function of genetic variants in non-coding regions. The intronic single nucleotide polymorphism (SNP) rs10994336 within the ANK3 has emerged as one of the most replicated risk variants for bipolar disorder (BD) in genome-wide association studies. Whether rs10994336 functions as a meQTL to mediate the association between genotype and phenotype remains unclear.

Method: A total of 154 patients with $\mathrm{BD}$ and 181 healthy controls $(\mathrm{HC})$ were recruited. The genotypes of rs 10994336 and methylation levels of CpG sites within ANK3 were tested. Executive functions were assessed using a computerized version of the Wisconsin Card Sorting Test (WCST).

Results: Bipolar disorder patients with the risk-T allele of rs10994336 scored lower on tests of executive function compared to homozygous CC carriers, after controlling for age, gender, and education level. No significant difference was found in $\mathrm{HC}$ individuals. The risk-T allele is associated with a lower methylation level of CpG site cg02172182 in $\mathrm{HC}$ after multiple corrections and replicated in the BD group in the same direction. Further mediation analysis revealed that the cg02172182 methylation significantly mediated the association between the polymorphism rs10994336 and PE index of WCST in patients with BD.

Conclusion: Our study suggests that BD-related genetic variant rs10994336 in ANK3 impacts executive functions by modulating ANK3 methylation, supporting the theory that methylation acts as a mediator between genotype and phenotype. 


\section{INTRODUCTION}

Bipolar Disorder (BD) is one of the most prevalent mental illnesses characterized by mood swings between episodes of mania or hypomania and depression (McIntyre et al., 2020). Genetic factors play a critical role in BD's etiology (Craddock and Sklar, 2013), supported by the estimated heritability as high as $\sim 80 \%$ (McGuffin et al., 2003). Family, twin, and adoption studies show that the lifetime prevalence of $\mathrm{BD}$ is $\sim 5-10 \%$ among firstdegree relatives (Lichtenstein et al., 2009) and $~ 40-70 \%$ among monozygotic twins of patients (Bertelsen et al., 1977; Kieseppä et al., 2004). Executive functions are higher-order cognitive processes that enable an individual to make complex decisions and process goal-oriented thoughts and behavior (Jurado and Rosselli, 2007; Miyake and Friedman, 2012). Executive function deficits have been well-reported in BD (Torres et al., 2007; Hellvin et al., 2012; Samamé et al., 2014; King et al., 2019) and their non-affected first-degree relatives (Bora et al., 2008; Kosger et al., 2015), suggesting that executive function deficits are inheritable features in BD (Frantom et al., 2008; Miskowiak et al., 2017). Considering that executive function is genetically less complicated than the disorder as a whole, it may help to understand the mechanism underlying $\mathrm{BD}$ by studying the effect of $\mathrm{BD}$-related genes on executive function.

The $\mathrm{T}$ allele of the intronic single nucleotide polymorphism (SNP) rs10994336 within the ANK3 gene are considered a risk allele for $\mathrm{BD}$ in genome-wide association studies (GWAS) (Ferreira et al., 2008; Scott et al., 2009; Psychiatric GWAS and Consortium Bipolar Disorder Working Group, 2011; Mühleisen et al., 2014; Stahl et al., 2019). Ankyrin 3 (ANK3) encodes Ankyrin-G, a cytoplasmic adaptor protein primarily located at the axonal initial segments and the nodes of Ranvier (Kordeli et al., 1995). Ankyrin-G plays many roles in neurotransmission, ion channel stabilization, cortical development (Durak et al., 2015), myelination (Ching et al., 1999), and neurogenesis in the adult brain (Paez-Gonzalez et al., 2011). AnkG hemizygous mice generated by the gene trapping approach demonstrated obvious cognitive impairment in an animal model study (Liu et al., 2017) and knockdown of ANK3 in drosophila is associated with memory deficits (Iqbal et al., 2013). Several studies have examined the association between rs10994336 polymorphism and executive functions in BD, a study of 49 patients with BD observed no effect of the rs10994336 genotype on executive function, memory, or general intelligence (Hori et al., 2014) and another study on 47 patients with BD either detected no association between the rs10994336 genotype and executive function (Ruberto et al., 2011). Since BD is a complex disorder and is influenced by a number of genetic variants and environmental exposures, the effect of a single risk allele on phenotype is small, thus, the larger the sample size is, the more power to detect the effect. Considering that the sample size included in those studies were relatively small, further larger sample studies are required to examine whether rs10994336 polymorphism could influence executive functions in BD.

Epigenetic modification including DNA methylation may play a critical role in BD's pathogenesis (Rakyan et al., 2011; Fries et al., 2016; Greenberg and Bourc'his, 2019). Both candidate studies and genome-wide methylation approaches using brain or blood samples have demonstrated promising methylation alterations associated with BD (Gürel et al., 2020). Many risk loci associated with $\mathrm{BD}$ are located in non-coding regions of the genome, suggesting that gene regulation plays a role in the disease pathology. Furthermore, different genotypes at a certain locus can alter DNA methylation pattern through allele-specific methylation (Schalkwyk et al., 2010; Bell et al., 2011). These sites are named methylation quantitative trait loci (meQTLs) and have been found throughout the genome in multiple tissues (Gibbs et al., 2010; Schalkwyk et al., 2010; Bell et al., 2011). Interestingly, it has been observed that meQTLs overlap with risk SNPs associated with diseases like BD, schizophrenia (SCZ), and Alzheimer's disease (Gamazon et al., 2013; Gaunt et al., 2016; Hannon et al., 2016). A previous study observed a significant overlap of meQTL between ancestral groups, developmental stages, and tissue types (Smith et al., 2014). Another study also suggests that DNA methylation contains a significant heritable component that is highly stable across the lifespan (Gaunt et al., 2016). These converging results shed light on the interpretation of genetic variants in non-coding regions that the influence of genetic variation on phenotype is potentially mediated through the allele-specific epigenetic processes.

In this study, we sought to study the associations between the rs10994336 variant of ANK3 and executive functions in patients with $\mathrm{BD}$ and healthy controls (HCs), and examine whether rs10994336 polymorphism functions as a meQTL and changes the site methylation to mediate the influences of rs10994336 on executive functions.

\section{MATERIALS AND METHODS}

\section{Participants}

A total of 335 participants between the age of 13 and 60 years were included in this study, including 154 with BD and 181 HCs. All participants with BD were recruited from the inpatient and outpatient service at the Department of Psychiatry, First Affiliated Hospital of China Medical University and Mental Health Center of Shenyang. The recruitment interval was from April 2011 to May 2019. In adult participants ( $\geq 18$ years old), the Axis I diagnosis (or its absence) were made by two experienced clinical psychiatrists using the Structured Clinical Interview Diagnostic (SCID) and Statistical Manual of Mental Disorder, Fourth Edition (DSM-IV). In participants younger than 18 years, the diagnosis (or absence) of Axis I Disorder were conducted using the Schedule for Affective Disorder and Schizophrenia for School-Age-Children-present (K-SADS-PL) by two experienced child psychiatrists. All the participants with $\mathrm{BD}$ in this study met the DSM-IV diagnostic criteria for BD and had no other Axis I Disorder. HC participants were recruited from Shenyang, China and surrounding cities by publicly posted advertisement. All HC participants had no current or lifetime history of an Axis I disorder or a history of psychotic, mood, or other Axis I Disorder in first-degree relatives, as determined from a detailed family history. Participants were excluded if they met any of the following criteria: (1) any history of major medical disease; (2) any history of moderate or severe head 
injury, head trauma, neurological disorder, or mental retardation; (3) lifetime substance/alcohol abuse or dependence; and/or (4) the presence of a concurrent and major physical illness that could lead to mood disorder symptoms. The current study was approved by the Ethics Committee of the First Affiliated Hospital of China Medical University (Shenyang China). Each participant provided a written informed consent after a complete description of the study. If their age were $<18$ years, they, as well as their parental/legal guardian, provided a written informed consent.

\section{Genotyping}

Venous blood samples were collected from the participants for DNA extraction pursuant to the standard procedures. Genotype information of rs10994336 was extracted from the results of the Illumina Global Screening Array-24 v1.0 BeadChip. Participants were further divided into two groups: a CC group (CC genotype; $91 \mathrm{BD}, 99 \mathrm{HC}$; mean age $=27.42 \pm 9.70$ years, $34.74 \%$ female) and risk T-carrier group (TT/CT genotypes; $63 \mathrm{BD}, 82 \mathrm{HC}$; mean age $=27.28 \pm 9.69$ years, $33.56 \%$ female). Genotype frequencies were consistent with Hardy-Weinberg equilibrium (HWE) expectations.

\section{DNA Methylation Data of ANK3}

DNA methylation level was assessed using the Infinium Human Methylation850 (850K) Beadchip array (Moran et al., 2016). To avoid batch effect, samples were processed in random order. All samples passed the quality assessment of assay performance requirements implemented in the Genome Studio software integrated controls dashboard. All DNA methylation levels were expressed as $\beta$-values, ranging from 0 to 1 , calculated as $M /(M+U)$, where $M$ is the signal from methylated beads and $U$ is the signal from unmethylated beads at the targeted CpG site and corrected for cell heterogeneity using the R package ChAMP (Tian et al., 2017). The genome coordinates provided by Illuminia (GRCh37/hg19) were used to map the independent methylation CpG site to a certain gene. Then, the $\beta$-values of all CpG sites within ANK3 were extracted for further analysis.

\section{Cognitive Measures}

The executive function was measured in 297 participants including $133 \mathrm{BD}$ and $164 \mathrm{HC}$ by the computerized version of the Wisconsin Card Sorting Test (WCST) (Heaton, 1981; Heaton et al., 1993), the most widely employed neuropsychological test for the assessment of executive function. This test requires subjects to alter response strategies and use previous irrelevant information to solve a problem. The WCST provides five indices as follows: correct response (CR), completed categories (CC), total errors (TE), perseverative errors (PE), and nonperseverative errors (NPE).

\section{Statistical Analyses Demographic and Clinical Data}

Independent two-sample $t$-tests or the chi-square tests were employed to investigate differences in the demographic and clinical data between the TT/TC group and CC group in participants with $\mathrm{BD}$ or $\mathrm{HC}$ participants. Continuous variables were presented as mean $\pm S D$. The threshold for significance level was defined as $P<0.05$.

\section{Association Between Rs10994336 and Cognition}

For all the participants who completed the WCST measures, we conducted one-way ANOVA with age, gender, and education years as covariates to explore the effect of genotype on the five indices in WCST. Statistical significance was set at two-tailed $P<0.05$.

\section{Association Between Rs10994336 and ANK3 Methylation Levels}

A total of $160 \mathrm{CpG}$ sites of ANK3 were included and all the corresponding $\beta$-values were calculated for each individual. The association between the TT/TC and CC groups of rs10994336 and each methylation level of the $160 \mathrm{CpG}$ sites was tested by a linear regression model adjusted with age and gender as covariates in $\mathrm{HC}$ participants and medication factor added as an extra covariate in the $\mathrm{BD}$ group. The significance level for the association analyses was set at $p<0.05$. A BenjaminHochberg false-discovery rate-corrected (FDR) correction was used to avoid type I error.

\section{Mediation Analysis}

To test whether the effect of rs10994336 genotype on cognition was potentially mediated by the methylation level of ANK3, we conducted mediation analysis on PE index using the PROCESS macro for SPSS, with a 5,000 bias-corrected bootstrap sample for significance testing. The mediation analysis was comprised of three steps of regression. In the first step, the outcome variable (PE index of WCST) was regressed on the independent variable (rs10994336 genotypes), which indicates the total effect of the predictor on the outcome variable. In the second step, the mediator variable (cg02172182 methylation) was regressed on the independent variable (rs10994336 genotypes), and in the final step, the outcome variable (PE index of WCST) was regressed on the mediator ( $\operatorname{cg} 02172182$ methylation) and the independent variable (rs10994336 genotypes). An indirect or mediating effect is supported when the significant association between the independent variable (rs10994336 genotypes) and outcome variable (PE index of WCST) diminishes after introducing a third variable, the mediator. In addition, age and gender were included as covariates in all mediation models and the study variables were standardized.

All the statistics analyses were conducted using the SPSS software except the association analysis between rs10994336 genotypes and methylation level of ANK3 which was performed by the $\mathrm{R}$ statistical package (http://cran.r-project.org) for multiple repeated tests.

\section{RESULTS}

\section{Demographic and Clinical Characteristics}

Demographic characteristics and clinical variables of patients with BD and HC subjects are presented in Table 1. There were no significant differences in age, gender, education level, or 
TABLE 1 | Demographic and clinical characteristics of different allele group in the BD and HC groups.

\begin{tabular}{|c|c|c|c|c|c|c|c|}
\hline & \multicolumn{2}{|c|}{$\mathrm{BD}(n=154)$} & \multicolumn{2}{|c|}{ HC $(n=181)$} & P1 & P2 & P3 \\
\hline Gender (M/F) & $19 / 44$ & $28 / 64$ & $30 / 52$ & $39 / 60$ & 0.971 & 0.699 & 0.134 \\
\hline Age (years) & $25.67 \pm 10.56$ & $26.85 \pm 9.67$ & $28.51 \pm 8.77$ & $27.95 \pm 9.69$ & 0.478 & 0.687 & 0.084 \\
\hline Education (years) & $12.33 \pm 3.19$ & $12.7 \pm 3.36$ & $14.74 \pm 3.3$ & $14.55 \pm 3.63$ & 0.502 & 0.725 & 0.131 \\
\hline Medication (Y/N) & $40 / 23$ & $61 / 30$ & NA & NA & 0.649 & NA & NA \\
\hline
\end{tabular}

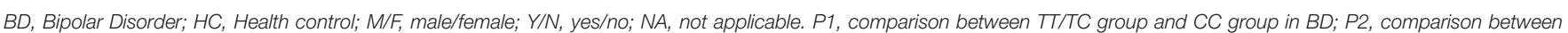
TT/TC group and CC group in HC; P3, comparison between BD and HC.

medication use between the $\mathrm{CC}$ and $\mathrm{TT} / \mathrm{TC}$ groups in $\mathrm{BD}$ or HC participants.

\section{Effects of Rs10994336 on Cognition}

The one-way ANOVA analysis was performed to investigate the effect of rs10994336 on cognition. The results were shown in Figure 1A for participants with BD and Figure 1B for HC participants, in which all five indices of WCST were significantly different between the T-allele carriers and homozygous CC individuals in $\mathrm{BD}$, while no significant difference was detected in $\mathrm{HC}$.

\section{Effects of Rs10994336 on ANK3 Methylation Levels}

We conducted an association study between rs10994336 and the methylation levels of ANK3 (160 CpG sites) in BD and $\mathrm{HC}$, respectively. After correcting for multiple tests, two CpG sites (cg02172182, P 0.017; cg093110194, P 0.011) showed a significant association with the genotype in $\mathrm{BD}$ participants, while eight CpG sites (cg05852740, P 0.003; cg06072426, P 0.1; cg11886031, P 0.045; cg12318342, P 0.034; cg02172182, P 0.047; cg 21429687 0.044; cg26504362, P 0.044; cg01186212, P 0.047) were associated significantly with the genotypes of rs10994336 in HC participants. The results of the association between the genotypes of rs10994336 and methylation level of CpG site cg02172182 were consistent in patients with BD (Figure 2A) and in HC (Figure 2B) individuals, where the TT/TC group had a lower methylation level of cg02172182 both in BD and HC.

\section{Mediation Effect}

Given the significant effect of polymorphism rs10994336 on executive functions in the BD group and that rs10994336 was detected to be a meQTL by influencing the methylation level of CpG site cg02172182 both in the HC and BD groups, we assessed whether the methylation level of cg02172182 mediated the association between rs10994336 and executive functions. The results showed that the methylation level of cg02172182 had a significant mediation effect on the association between ANK3 rs10994336 genotypes and PE index of WCST in the $\mathrm{BD}$ group (Figure 3). In step one of the mediation analysis, rs10994336 genotypes significantly predicted the PE index of WCST (path C), and rs10994336 genotypes also predicted cg02172182 methylation (path A) in step two. In the final step, we found that the predictive effect of rs10994336 genotypes on PE (index of WCST) became less significant after introducing cg02172182 methylation as a mediator (path C' < path C), thus demonstrating that cg02172182 methylation partially mediated the association between rs10994336 genotypes and PE index of WCST.

\section{DISCUSSION}

In the present study, we found that the risk T-allele carriers of rs10994336 showed a worse WCST performance compared to those with homozygous CC genotype in the $\mathrm{BD}$ group. Moreover, rs10994336 affects the methylation level of specific CpG site within ANK3 as a meQTL in the BD and $\mathrm{HC}$ groups, which mediates the effect of rs10994336 on executive functions. To the best of our knowledge, it is the first study to document the mediation effect of ANK3 methylation between BD-related genetic variant rs10994336 within ANK3 and executive functions in BD.

Our results suggest that the risk allele of rs10994336 of ANK3 is associated with a poor executive performance in patients with $\mathrm{BD}$ after adjusting for age, gender, and education. This is consistent with the result of a previous study conducted among 173 patients with first-episode psychosis showing that the polymorphism rs1938526 of ANK3 gene, which was in high linkage disequilibrium with rs10994336, was associated with cognitive domains of reasoning and problem solving, though at a marginal significance (Cassidy et al., 2014). Moreover, the loss of association between rs10994336 and executive functions in the HC group was consistent with two previous studies which detected no effect of this SNP on measures of executive function either in HC individuals (Hori et al., 2014) or in healthy males (Roussos et al., 2011). However, two studies on BD failed to examine the association between rs10994336 and executive function, memory, or general intelligence (Ruberto et al., 2011; Hori et al., 2014). It should be noted that the sample size included in those studies were relatively small which reduced the statistical power to test the association. Considering the complex interplay between genetic and environmental factors in the etiology contributing to $\mathrm{BD}$, we speculate that the association between rs10994336 and executive function is predominant in 
A

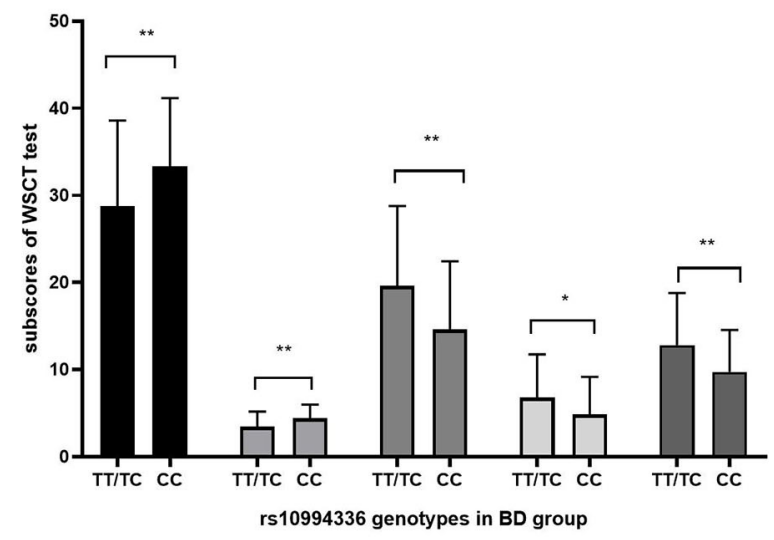

B

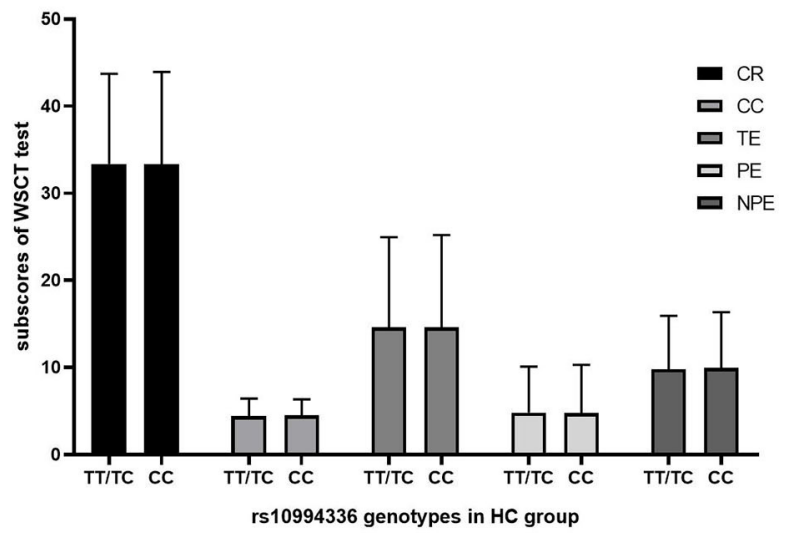

FIGURE 1 | The association between genotypes of rs10994336 and cognition. Comparisons were made for five indices of WSCT between rs10994336 T-allele carriers and C-allele homozygotes in patients with BD (A) and in HC (B). WCST, Wisconsin Card Sorting Test; BD, bipolar disorder; HC, healthy control; CR, correct response; CC, completed categories; TE, total errors; PE, perseverative errors; NPE, non-perseverative errors. Error bars represent standard errors of the means. ${ }^{*} P$ $<0.05,{ }^{\star \star} P<0.01$ (according to the ANCOVA controlling for age, gender, and education).

A

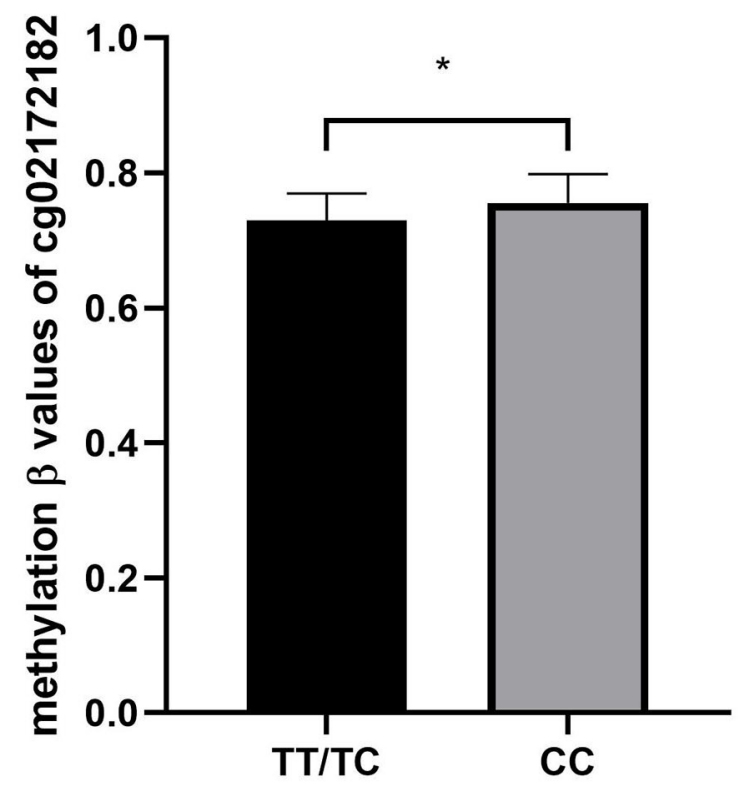

rs10994336 genotypes in BD group
B

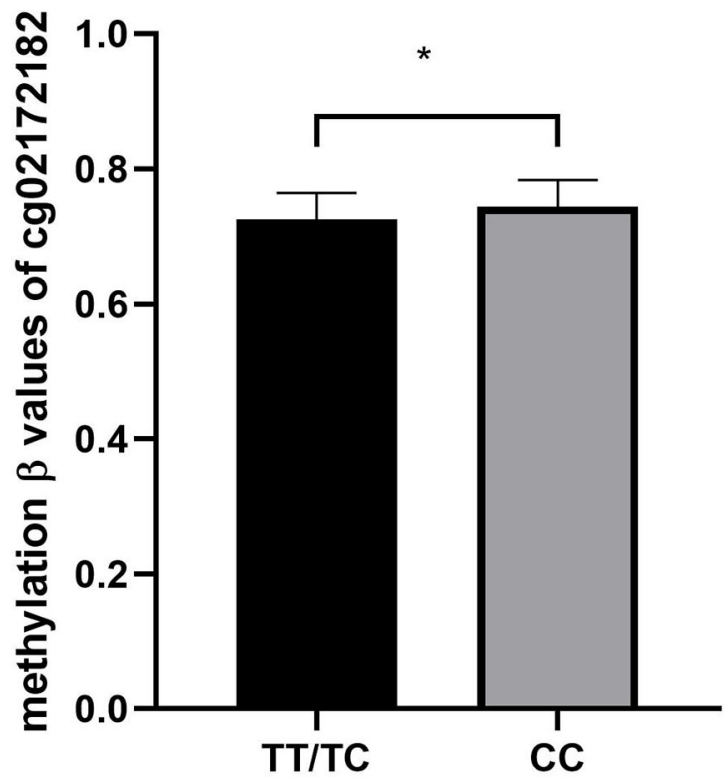

rs10994336 genotypes in HC group

FIGURE 2 | The association between genotypes of rs10994336 and the methylation level at CpG site cg2172182 within ANK3 in patients with BD (A) and in HC (B). $\mathrm{BD}$, bipolar disorder; $\mathrm{HC}$, healthy control. ${ }^{*} P<0.05$ after adjusting for multiple tests by the Benjamini-Hochberg false-discovery rate-corrected (FDR) correction.

patients with $\mathrm{BD}$, who have a predisposition to severe cognitive deficits due to the complex pathophysiological processes induced by genetic factors. Studies with larger samples are needed to test whether these findings can be replicated.
The polymorphism rs10994336 is located in the intron of ANK3 gene, and its function was unclear until now. We tested whether this SNP affected the methylation level of ANK3. Considering variables such as age, gender, and medication 


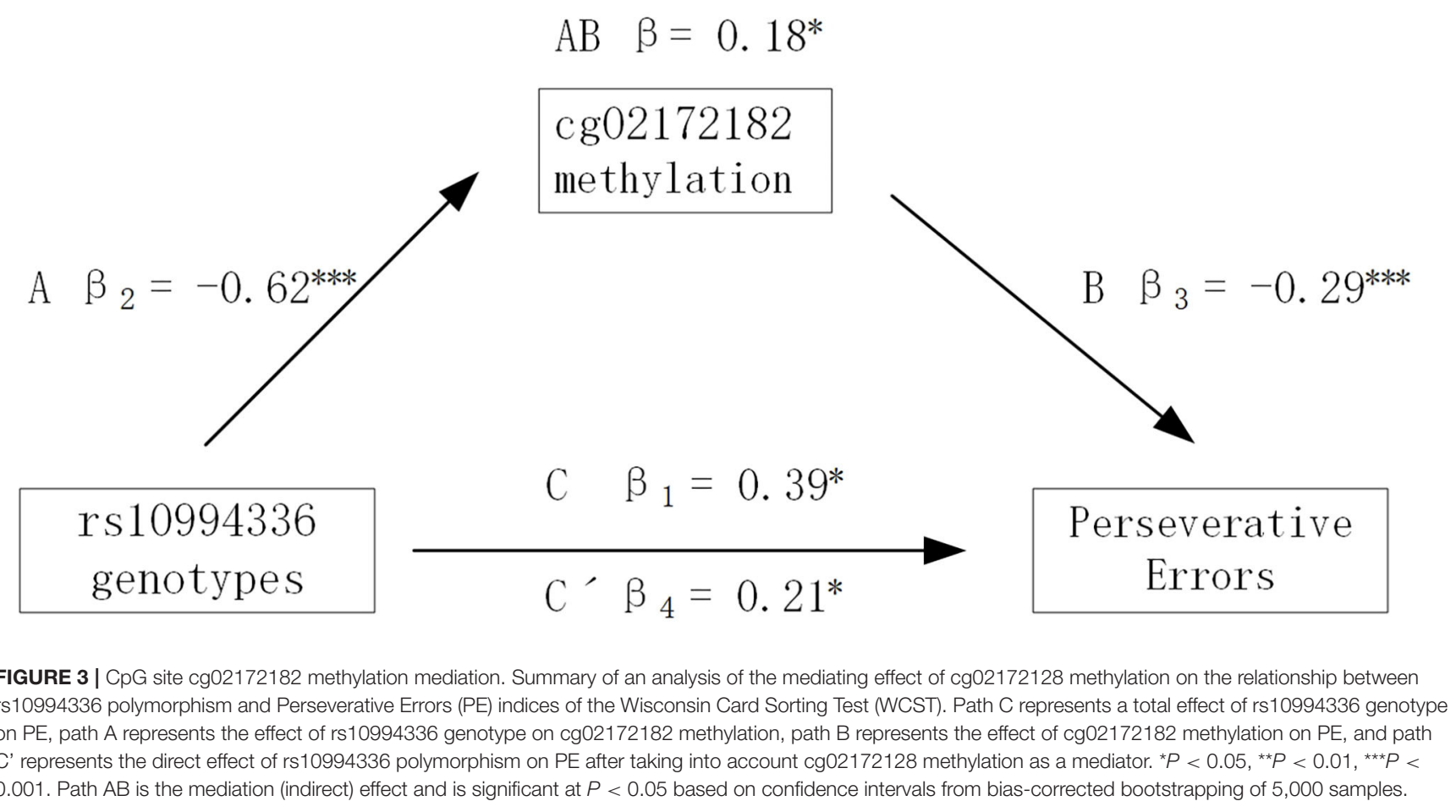

use were believed to influence DNA methylation (Burghardt et al., 2020; Gardea-Resendez et al., 2020; Webb et al., 2020), we add these confounding factors in our linear regression model as covariates to explore the allele-associated methylation. After adjusting these confounders, the effect of rs10994336 polymorphism on methylation of the CpG site cg02172182 within the gene body of ANK3 was observed in $\mathrm{HC}$ and replicated in patients with $\mathrm{BD}$ in the same direction where the risk T-allele carriers presented a reduced methylation relative to homozygous CC participants, suggesting that the effect of genotype on methylation is not driven by the disease and rs10994336 could be referred to as a reliable meQTL. Cg02172182 locates at the gene body region of ANK3 and $\sim 1.5 \mathrm{~kb}$ upstream of rs10994336, which functions by cisacting meQTL that can act over a range of distances, from a few base pairs to distances of over $500 \mathrm{~kb}$. Our mediation analysis model in $\mathrm{BD}$ supports the theory that methylation acts as a mediator between genotype and phenotype, as the effect of rs10994336 polymorphism on PE was significantly mediated by the methylation of cg02172182. Although the methylation patterns are highly heterogeneous between different tissue types and at different life stages, genetic effects on methylation are found to be rather stable across life course and different tissue types (Smith et al., 2014; Gaunt et al., 2016). A previous study identified separately and compared genome-wide meQTLs from brain prefrontal cortex, whole blood, and saliva and observed significant overlap of meQTLs among tissues (Smith et al., 2014). Moreover, another study also implicated that the DNA methylation status of many CpG sites in the brain were mirrored in the blood and that common meQTLs were also detected between these two tissues (Gaunt et al., 2016). These converging findings support the theory that blood tissue could be used as a surrogate tissue to explore disease relevant processes within the brain. In addition, multiple strands of evidence indicate that DNA methylation plays an important role in several neurobiological processes including neurogenesis (Costello, 2003), neural plasticity (Dulac, 2010), and the formation and maintenance of memories (Halder et al., 2016), which in turn impacts on executive function. Prompted by these findings, it is valid to speculate that the function of rs10994336 as a meQTL in whole blood tissue may mirror the function in brain tissues which influences the methylation of ANK3 gene and further results in executive function impairment in patients with $\mathrm{BD}$.

There are several limitations to the present study. First, the sample size of our study did not have sufficient power to detect the difference of frequencies of rs10994336, or the difference of methylation of ANK3 between BD and HC. Since rs10994336 is a well-established BD risk variant, the difference of genotype or allele frequencies between BD and control will lead to the difference of methylation of this gene, which will have an effect on brain function. This may explain why we only detected the association of rs10994336 with executive functions in BD. Second, the actual effect of rs10994336 polymorphism on ANK3 methylation in brain tissues in which the pathological processes for psychiatric 
disorder are likely involved could not be directly observed in our study, since brain tissues are not readily accessible in living patients. However, research findings indicate a common overlap of meQTLs between different tissue types including blood and brain tissues. Third, we did not stratify our samples according to those confounding factors such as age, medication use, and disease status (manic, depressive, and remission) which could affect DNA methylation considering that our sample size was not large enough. However, age and medication use were adjusted in our linear regression models and the effect of rs10994336 polymorphism on cg121722182 methylation remained significant. Future studies with larger samples could further exclude those confounders. Finally, a potential limitation is that the precise mechanism by which methylation of $\operatorname{cg} 121722182$ influences executive functions is unclear since it locates at the gene body of ANK3 while the role of methylation in gene bodies is not well-established, but some evidence indicates that methylation in gene bodies is associated with the activation of genes in contrast to the methylation observed in promoter regions which are generally considered to be involved in the content of silence of genes (Greenberg and Bourchis, 2019). Future studies are needed to address this question.

In summary, using executive functions as a highly heritable endophenotype and combining genetic and epigenetic data, we found that allelic variation of rs10994336 in ANK3 impacts executive functions by moderating methylation, supporting the theory that methylation acts as a mediator between genotype and phenotype. This provides insights into the mechanism of how intron risk variants associated with $\mathrm{BD}$ contribute to disease susceptibility.

\section{REFERENCES}

Bell, J. T., Pai, A. A., Pickrell, J. K., Gaffney, D. J., Pique-Regi, R., Degner, J. F., et al. (2011). DNA methylation patterns associate with genetic and gene expression variation in HapMap cell lines. Genome Biol. 12:R10. doi: 10.1186/gb-2011-12-1-r10

Bertelsen, A., Harvald, B., and Hauge, M. (1977). A Danish twin study of manicdepressive disorder. Br. J. Psychiatry 130, 330-351. doi: 10.1192/bjp.130.4.330

Bora, E., Vahip, S., Akdeniz, F., Ilerisoy, H., Aldemir, E., and Alkan, M. (2008). Executive and verbal working memory dysfunction in first-degree relatives of patients with bipolar disorder. Psychiatry Res. 161, 318-324. doi: 10.1016/j.psychres.2007.09.002

Burghardt, K. J., Khoury, A. S., Msallaty, Z., Yi, Z., and Seyoum, B. (2020). Antipsychotic medications and DNA methylation in schizophrenia and bipolar disorder: a systematic review. Pharmacotherapy 40, 331-342. doi: 10.1002/phar.2375

Cassidy, C., Buchy, L., Bodnar, M., Dell'Elce, J., Choudhry, Z., Fathalli, F., et al. (2014). Association of a risk allele of ANK3 with cognitive performance and cortical thickness in patients with first-episode psychosis. J. Psychiatry Neurosci. 39, 31-39. doi: 10.1503/jpn.120242

Ching, W., Zanazzi, G., Levinson, S. R., and Salzer, J. L. (1999). Clustering of neuronal sodium channels requires contact with myelinating Schwann cells. J. Neurocytol. 28, 295-301. doi: 10.1023/A:1007053411667

Costello, J. F. (2003). DNA methylation in brain development and gliomagenesis. Front Biosci. 8:s175-184. doi: 10.2741/1027

\section{DATA AVAILABILITY STATEMENT}

The raw data supporting the conclusions of this article will be made available by the authors, without undue reservation.

\section{ETHICS STATEMENT}

The studies involving human participants were reviewed and approved by Ethics Committee of First Affiliated Hospital of China Medical University. Written informed consent to participate in this study was provided by the participants' legal guardian/next of kin.

\section{AUTHOR CONTRIBUTIONS}

YT and FW contributed to the conception and design of the study and provided the technical support. LT performed the data analyses and wrote the manuscript. LT, JL, YW, YZ, JD, and YC contributed to acquisition of data and manuscript preparation. XG helped perform the analysis with constructive discussions. All authors contributed to the article and approved the submitted version.

\section{FUNDING}

National Science Fund for Distinguished Young Scholars (81725005 to FW), Liaoning Education Foundation (Pandeng Scholar to FW), National Key Research Development Program (2018YFC1311604 and 2016YFC1306900 to YT), Innovation Team Support Plan of Higher Education of Liaoning Province (LT2017007 to FW), and Major Special Construction Plan of China Medical University (3110117059 and 3110118055 to FW).

Craddock, N., and Sklar, P. (2013). Genetics of bipolar disorder. Lancet 381, 1654-1662. doi: 10.1016/S0140-6736(13) 60855-7

Dulac, C. (2010). Brain function and chromatin plasticity. Nature 465, 728-735 doi: $10.1038 /$ nature09231

Durak, O., de Anda, F. C., Singh, K. K., Leussis, M. P., Petryshen, T. L., Sklar, P., et al. (2015). Ankyrin-G regulates neurogenesis and Wnt signaling by altering the subcellular localization of $\beta$-catenin. Mol. Psychiatry 20, 388-397. doi: $10.1038 / \mathrm{mp} .2014 .42$

Ferreira, M. A. R., O’Donovan, M. C., Meng, Y. A., Jones, L. R., Ruderfer, D. M., Jones, L., et al. (2008). Collaborative genome-wide association analysis supports a role for ANK3 and CACNA1C in bipolar disorder. Nat. Genet. 40, 1056-1058. doi: 10.1038/ng.209

Frantom, L. V., Allen, D. N., and Cross, C. L. (2008). Neurocognitive endophenotypes for bipolar disorder. Bipolar Disord. 10, 387-399. doi: 10.1111/j.1399-5618.2007.00529.x

Fries, G. R., Li, Q., McAlpin, B., Rein, T., Walss-Bass, C., Soares, J. C., et al. (2016). The role of DNA methylation in the pathophysiology and treatment of bipolar disorder. Neurosci. Biobehav. Rev. 68, 474-488. doi: 10.1016/j.neubiorev.2016.06.010

Gamazon, E. R., Badner, J. A., Cheng, L., Zhang, C., Zhang, D., Cox, N. J., et al. (2013). Enrichment of cis-regulatory gene expression SNPs and methylation quantitative trait loci among bipolar disorder susceptibility variants. Mol. Psychiatry 18, 340-346. doi: 10.1038/mp. 2011.174 
Gardea-Resendez, M., Kucuker, M. U., Blacker, C. J., Ho, A. C., Croarkin, P. E., Croarkin, M. A., et al. (2020). Dissecting the epigenetic changes induced by non-antipsychotic mood stabilizers on schizophrenia and affective disorder: a systematic review. Front. Pharmacol. 11:467. doi: 10.3389/fphar.2020. 00467

Gaunt, T. R., Shihab, H. A., Hemani, G., Min, J. L., Woodward, G., Lyttleton, O., et al. (2016). Systematic identification of genetic influences on methylation across the human life course. Genome Biol. 17:61. doi: 10.1186/s13059-016-0926-z

Gibbs, J. R., Van Der Brug, M. P., Hernandez, D. G.,Traynor, B. J., Nalls, M. A., Lai, S. L., et al. (2010). Abundant quantitative trait loci exist for DNA methylation and gene expression in human brain. PLoS Genet. 6:e1000952. doi: 10.1371/journal.pgen.1000952

Greenberg, M. V. C., and Bourc'his, D. (2019). The diverse roles of DNA methylation in mammalian development and disease. Nat. Rev. Mol. Cell Biol. 20, 590-607. doi: 10.1038/s41580-019-0159-6

Gürel, Ç., Kuşçu, G. C., Yavaşoglu, A., and Biray Avci, Ç. (2020). The clues in solving the mystery of major psychosis: the epigenetic basis of schizophrenia and bipolar disorder. Neurosci. Biobehav. Rev. 113, 51-61. doi: 10.1016/j.neubiorev.2020.03.005

Halder, R., Hennion, M., Vidal, R. O., Shomroni, O., Rahman, R. U., Rajput, A., et al. (2016). DNA methylation changes in plasticity genes accompany the formation and maintenance of memory. Nat. Neurosci. 19, 102-110. doi: $10.1038 / \mathrm{nn} .4194$

Hannon, E., Spiers, H., Viana, J., Pidsley, R., Burrage, J., Murphy, T. M., et al. (2016). Methylation QTLs in the developing brain and their enrichment in schizophrenia risk loci. Nat. Neurosci. 19, 48-54. doi: 10.1038/nn.4182

Heaton, R. K. (1981). The Wisconsin Card Sorting Test Manual. Odessa, FL: Psychological Assessment Resources.

Heaton, R. K., Chelune, G. J., Talley, J. L., Kay, G. G., and Curtiss, G. (1993). Wisconsin Card Sorting Test Manual-Revised and Expanded. Odessa, FL: Psychological Assessment Resources.

Hellvin, T., Sundet, K., Simonsen, C., Aminoff, S. R., Lagerberg, T. V., Andreassen, O. A., et al. (2012). Neurocognitive functioning in patients recently diagnosed with bipolar disorder. Bipolar Disord. 14, 227-238. doi: 10.1111/j.1399-5618.2012.01004.x

Hori, H., Yamamoto, N., Teraishi, T., Waryah, A. M., Zahoor, M. Y., Besseling, J. A., et al. (2014). Cognitive effects of the ANK3 risk variants in patients with bipolar disorder and healthy individuals. J. Affect. Disord. 158, 90-96. doi: 10.1016/j.jad.2014.02.008

Iqbal, Z., Vandeweyer, G., Van Der Voet, M., Waryah, A. M., Zahoor, M. Y., Besseling, J. A., et al. (2013). Homozygous and heterozygous disruptions of ANK3: at the crossroads of neurodevelopmental and psychiatric Disorder. Hum. Mol. Genet. 22, 1960-1970. doi: 10.1093/hmg/ddt043

Jurado, M. B., and Rosselli, M. (2007). The elusive nature of executive functions: a review of our current understanding. Neuropsychol. Rev. 17, 213-233. doi: $10.1007 /$ s11065-007-9040-z

Kieseppä, T., Partonen, T., Haukka, J., Kaprio, J., and Lönnqvist, J. (2004). High concordance of bipolar I disorder in a nationwide sample of twins. Am. J. Psychiatry 161, 1814-1821. doi: 10.1176/ajp.161.10.1814

King, S., Stone, J. M., Cleare, A., and Young, A. H. (2019). A systematic review on neuropsychological function in bipolar disorder type I and II and subthreshold bipolar disorder-something to think about. CNS Spectr. 24, 127-143. doi: 10.1017/S1092852918001463

Kordeli, E., Lambert, S., Bennett, V., and Ankyrin, G. (1995). A new ankyrin gene with neural-specific isoforms localized at the axonal initial segment and node of Ranvier. J. Biol. Chem. 270, 2352-2359. doi: 10.1074/jbc.270. 5.2352

Kosger, F., Essizoglu, A., Baltacioglu, M., Ulkgun, N., and Yenilmez, C. (2015). Executive function in parents of patients with familial versus sporadic bipolar disorder. Compr. Psychiatry 61, 36-41. doi: 10.1016/j.comppsych.2015. 05.013

Lichtenstein, P., Yip, B. H., Björk, C., Pawitan, Y., Cannon, T. D., Sullivan, P. F., et al. (2009). Common genetic determinants of schizophrenia and bipolar disorder in Swedish families: a population-based study. Lancet 373, 234-239. doi: 10.1016/S0140-6736(09)60072-6

Liu, C., Zhang, L., Wu, J., Sui, X. L., Xu, Y. F., Huang, L., et al. (2017). AnkG hemizygous mice present cognitive impairment and elevated anxiety/depressive-like traits associated with decreased expression of GABA receptors and postsynaptic density protein. Exp. Brain Res. 235, 3375-3390. doi: 10.1007/s00221-017-5056-7

McGuffin, P., Rijsdijk, F., Andrew, M., Sham, P., Katz, R., and Cardno, A. (2003). The heritability of bipolar affective disorder and the genetic relationship to unipolar depression. Arch. Gen. Psychiatry 60, 497-502. doi: 10.1001/archpsyc.60.5.497

McIntyre, R. S., Berk, M., Brietzke, E., Goldstein, B. I., López-Jaramillo, C., Kessing, L. V., et al. (2020). Bipolar disorder. Lancet 396, 1841-1856. doi: 10.1016/S0140-6736(20)31544-0

Miskowiak, K. W., Kjærstad, H. L., Meluken, I., Petersen, J. Z., and Maciel, B. R. (2017). The search for neuroimaging and cognitive endophenotypes: a critical systematic review of studies involving unaffected first-degree relatives of individuals with bipolar disorder. Neurosci. Biobehav. Rev. 73, 1-22. doi: 10.1016/j.neubiorev.2016.12.011

Miyake, A., and Friedman, N. P. (2012). The nature and organization of individual differences in executive functions: four general conclusions. Curr. Dir. Psychol. Sci. 21, 8-14. doi: 10.1177/0963721411429458

Moran, S., Arribas, C., and Esteller, M. (2016). Validation of a DNA methylation microarray for $850,000 \mathrm{CpG}$ sites of the human genome enriched in enhancer sequences. Epigenomics 8, 389-399. doi: 10.2217/epi. 15.114

Mühleisen, T. W., Leber, M., Schulze, T. G., Strohmaier, J., Degenhardt, F., Treutlein, J., et al. (2014). Genome-wide association study reveals two new risk loci for bipolar disorder. Nat. Commun.5:3339. doi: 10.1038/ncomm s4339

Paez-Gonzalez, P., Abdi, K., Luciano, D., Liu, Y., Soriano-Navarro, M., Rawlins, E., et al. (2011). Ank3-dependent SVZ niche assembly is required for the continued production of new neurons. Neuron 71, 61-75. doi: 10.1016/j.neuron.2011.05.029

Psychiatric GWAS and Consortium Bipolar Disorder Working Group. (2011). Large-scale genome-wide association analysis of bipolar disorder identifies a new susceptibility locus near ODZ4. Nat. Genet. 43, 977-983. doi: 10.1038/ng.943

Rakyan, V. K., Down, T. A., Balding, D. J., and Beck, S. (2011). Epigenome-wide association studies for common human diseases. Nat. Rev. Genet. 12, 529-541. doi: $10.1038 / \operatorname{nrg} 3000$

Roussos, P., Giakoumaki, S. G., Georgakopoulos, A., Robakis, N. K., and Bitsios, P. (2011). The CACNA1C and ANK3 risk alleles impact on affective personality traits and startle reactivity but not on cognition or gating in healthy males. Bipolar Disord. 13, 250-259. doi: 10.1111/j.1399-5618.2011. 00924.x

Ruberto, G., Vassos, E., Lewis, C. M., Tatarelli, R., Girardi, P., Collier, D., et al. (2011). The cognitive impact of the ANK3 risk variant for bipolar disorder: initial evidence of selectivity to signal detection during sustained attention. PLOS ONE 6:e16671. doi: 10.1371/journal.pone. 0016671

Samamé, C., Martino, D. J., and Strejilevich, S. A. (2014). Longitudinal course of cognitive deficits in bipolar disorder: a meta-analytic study. J. Affect. Disord. 164, 130-138. doi: 10.1016/j.jad.2014.04.028

Schalkwyk, L. C., Meaburn, E. L., Smith, R., Dempster, E. L., Jeffries, A. R., Davies, M. N., et al. (2010). Allelic skewing of dna methylation is widespread across the genome. Am. Hum. Genet. 86, 196-212. doi: 10.1016/j.ajhg.2010. 01.014

Scott, L. J., Muglia, P., Kong, X. Q., Guan, W., Upmanyu, R., Tozzi, F., et al. (2009). Genome-wide association and meta-analysis of bipolar disorder in individuals of European ancestry. Proc. Natl. Acad. Sci. U.S.A. 106, 7501-7506. doi: 10.1073/pnas.0813386106

Smith, A. K., Kilaru, V., Kocak, M., Almli, L. M., Mercer, K. B., Ressler, K. J., et al. (2014). Methylation quantitative trait loci (meQTLs) are consistently detected across ancestry, developmental stage, and tissue type. BMC Genomics 15:145. doi: 10.1186/1471-2164-15-145

Stahl, E. A., Breen, G., Forstner, A. J., McQuillin, A., Ripke, S., Trubetskoy, V., et al. (2019). Genome-wide association study identifies 30 loci associated with bipolar disorder. Nat. Genet. 51, 793-803. doi: 10.1038/s41588-0190397-8

Tian, Y., Morris, T. J., Webster, A. P., Yang, Z., Beck, S., Feber, A., et al. (2017). ChAMP: updated methylation analysis pipeline for Illumina 
BeadChips. Bioinformatics 33, 3982-3984. doi: 10.1093/bioinformatics/ btx513

Torres, L. J., Boudreau, V. G., and Yatham, L. N. (2007). Neuropsychological functioning in euthymic bipolar disorder: a meta-analysis. Acta Psychiatr. Scand. Suppl. 2007, 17-26. doi: 10.1111/j.1600-0447.2007. 01055.x

Webb, L. M., Phillips, K. E., Ho, M. C., Veldic, M., and Blacker, C. J. (2020). The relationship between DNA methylation and antidepressant medications: a systematic review. Int. J. Mol. Sci. 21:826. doi: 10.3390/ijms21030826

Conflict of Interest: The authors declare that the research was conducted in the absence of any commercial or financial relationships that could be construed as a potential conflict of interest.
Publisher's Note: All claims expressed in this article are solely those of the authors and do not necessarily represent those of their affiliated organizations, or those of the publisher, the editors and the reviewers. Any product that may be evaluated in this article, or claim that may be made by its manufacturer, is not guaranteed or endorsed by the publisher.

Copyright (c) 2021 Tang, Liu, Zhu, Duan, Chen, Wei, Gong, Wang and Tang. This is an open-access article distributed under the terms of the Creative Commons Attribution License (CC BY). The use, distribution or reproduction in other forums is permitted, provided the original author(s) and the copyright owner(s) are credited and that the original publication in this journal is cited, in accordance with accepted academic practice. No use, distribution or reproduction is permitted which does not comply with these terms. 